\title{
BMJ Open Potentially inappropriate prescribing in dementia: a state-of-the-art review since 2007
}

\author{
Joao Delgado (1), Kirsty Bowman, Linda Clare
}

To cite: Delgado J, Bowman K, Clare L. Potentially inappropriate prescribing in dementia: a state-of-the-art review since 2007. BMJ Open 2020;10:e029172. doi:10.1136/ bmjopen-2019-029172

- Prepublication history and additional material for this paper are available online. To view these files, please visit the journal online (http://dx.doi. org/10.1136/bmjopen-2019029172).

Received 16 January 2019 Revised 12 November 2019 Accepted 13 November 2019

Check for updates

(C) Author(s) (or their employer(s)) 2020. Re-use permitted under CC BY-NC. No commercial re-use. See rights and permissions. Published by BMJ.

College of Medicine and Health, University of Exeter, Exeter, UK

Correspondence to

Dr Joao Delgado;

j.correa-delgado@exeter.ac.uk

\section{ABSTRACT}

Objectives Dementia frequently occurs alongside comorbidities. Coexisting conditions are often managed with multiple medications, leading to increased risk of potentially inappropriate medication and adverse drug reactions. We aimed to estimate prevalence of, and identify factors reported to be associated with, potentially inappropriate prescribing (PIP) for older individuals diagnosed with dementia.

Design We used a state-of-the-art review approach, selecting papers written in English and published from 2007 to January 2018. Publications were retrieved from Scopus and Web of Science databases. Inclusion criteria included a formal diagnosis of dementia, a formal classification of PIP and reported prevalence of PIP as an outcome. Random effects models were used to provide a pooled estimate of prevalence of PIP. The Appraisal tool for Cross-Sectional Studies (AXIS tool) was used to assess bias in the included studies.

Results The bibliographic search yielded 221 citations, with 12 studies meeting the inclusion criteria. The estimates of PIP prevalence for people living with dementia ranged from $14 \%$ to $64 \%$. Prevalence was $31 \%$ (95\% Cl 9 to 52 ) in the community, and $42 \%$ (95\% Cl 30 to 55) in nursing/care homes. PIP included prescribing likely related to dementia (eg, hypnotics and sedative and cholinesterase inhibitors) and prescribing related to treatment of comorbidities (eg, cardiovascular drugs and non-steroidal anti-inflammatory medication). Higher levels of comorbidity were associated with increased risk of PIP; however, only one study investigated associations with specific comorbidities of dementia.

Conclusion PIP remains a significant issue in healthcare management for people living with dementia. Higher levels of comorbidity are associated with increased prevalence of PIP, but the specific conditions driving this increase remain unknown. Further work is necessary to investigate PIP related to the presence of common comorbidities in patients living with dementia.

\section{INTRODUCTION}

Dementia defines a group of conditions involving irreversible neurodegenerative disease, leading to changes in cognition, communication and functional ability. ${ }^{1}$ The most common form of dementia is Alzheimer's disease $(\mathrm{AD})$, followed by cerebrovascular dementia (VaD), mixed $\mathrm{AD}$ and $\mathrm{VaD}$,

\section{Strengths and limitations of this study}

This study collates up-to-date prevalence estimates of potentially inappropriate prescribing, comorbidities and medications in people living with dementia.

- The structured approach used to identify relevant studies was applied to the most widely used databases.

- The inclusion criteria aimed to ensure high-quality research was included in this review.

- These criteria, however, may have restricted the number of studies included in our final selection.

- Study heterogeneity means that prevalence estimates must be viewed as a guide for discussion, and not as accurate estimates.

and other dementias. ${ }^{2}{ }^{3}$ In the UK, prevalence of dementia is estimated at 2\%-3\% in those aged 65-74 years, but increases to $30 \%-50 \%$ in those aged 85 years and older. ${ }^{45}$ A diagnosis of dementia often occurs alongside other conditions common in ageing individuals, such as hypertension (68\%), chronic kidney disease (stages 3-5; 30\%), coronary heart disease $(29 \%)$ and diabetes $(20 \%))^{5}$ Thus, management of dementia in old age often takes place in the context of managing additional comorbidities. ${ }^{6-9}$

The management of multiple conditions carries with it the prescription of multiple medications. Polypharmacy, often defined as the concurrent use of multiple (eg, five or more) prescription drugs by a patient, is common in people living with dementia, who have an estimated average of 5-10 prescriptions at any one time. ${ }^{10} 11$ Polypharmacy in itself is not always inappropriate but adds the challenge of managing the possible adverse side effects of each medication and the effects of potential drug interactions. This can be quite complex and as a result individuals prescribed several medications are at greater risk of potentially inappropriate prescribing (PIP). ${ }^{12} 13$ PIP is defined as the use of medicines that pose more risk than benefit, 
particularly where safer alternatives exist. ${ }^{14}$ Adverse drug reactions (ADR), in turn, lead to increased risk of hospital admission and mortality, and higher healthcare costs. $^{131516}$

Multiple tools are available to help identify PIP. ${ }^{17}$ These tools evaluate prescribing using explicit (criterion-based) or implicit (judgement-based) approaches to identify instances where the presence of medical conditions and medications may lead to an increase in the risk of adverse drug reactions. ${ }^{14} 18$ Most tools are developed with a specific interest in older individuals, and differences are often associated with the differences in care and medication available in different countries or regions, for example, the USA and the European Union (EU). These are best exemplified by Beer's criteria developed for the US market and the screening tool for potentially inappropriate prescribing in older people that can alert doctors to the correct treatment (STOPP/START criteria) developed for the EU market. ${ }^{19} 20$ An exception to this is the set of criteria developed by Holmes et al, which was produced specifically for people living with dementia. $^{21}$

Issues associated with comorbidities and PIP are not unique to people living with dementia, and studies have been published investigating the effects of PIP in older people in acute and long-term care, as well as those living in the community. ${ }^{142-25}$ However, few studies have focused on the appropriateness of prescribing, particularly in the presence of comorbidities, in people living with dementia. ${ }^{22}{ }^{26}$ Individuals living with dementia are often excluded from large trials due to their age, short life expectancy and difficulty communicating symptoms. ${ }^{27} 28$ The sparse information available on multimorbidity, polypharmacy and inappropriate prescribing means that decisions are often made based on studies targeting healthier individuals-evidence that may not be applicable to those with dementia. Consequentially, navigating medication management for people with dementia remains a challenge for clinicians. ${ }^{27} 29$

Here, we review research articles published in peerreviewed journals in the past 10 years that focus on PIP in individuals living with dementia. We place specific focus on the common comorbidities of dementia and the prescribed medications most commonly associated with PIP and adverse drug reactions. Our objective is to estimate the extent of PIP in people with dementia, and understand the role of treatment of comorbidities in determining prescribing quality. In order to manage the scope of this review, we focused on studies produced in North America, Western Europe and Australasia. We aim to gather existing evidence and highlight gaps in the information available to support improvements to pharmacological management of multiple conditions in the context of dementia.
METHODS

\section{Search strategy}

This is a state-of-the-art review, involving a structured search of current literature. ${ }^{30}$ This is a condensed subtype of literature review, providing a comprehensive analysis of current literature (eg, from the past 10 years) but a condensed approach to reviewing the studies. The objective of such reviews is not to perform an exhaustive review of literature but rather to summarise current trends and identify research priorities of interest. ${ }^{30}$ We developed a strategy designed to facilitate repeatability and future updates. Search terms were defined for identifying quantitative studies assessing prevalence of PIP in individuals diagnosed with dementia. Search terms for identifying dementia-related studies were retrieved from a Cochrane review on treatment of depression in dementia. ${ }^{31}$ The remainder of the search terms were developed for the Scopus database. Search terms were then adapted for Web of Science. Scopus is a database providing access to Science, Technical and Medical (STM) journal articles, including coverage of Medline, Embase and Compendex. As our focus was on identifying recently published studies, the search was limited to the period 2007 to October 2018. The search terms were as follows:

- Title, key words or abstract: dement* OR alzheimer* OR lewy OR cjd OR jcd OR ad OR add OR dlb OR huntington* OR frontotemporal

- AND in the title, key words or abstract: "inappropriate prescribing" OR "inappropriate prescription" OR "inappropriate medication"

- AND in the title, key words or abstract: prevalence OR incidence OR percentage OR rate OR rates

- AND in the title, key words or abstract cohort OR prospective OR retrospective OR "cross sectional" OR "cross-sectional"

- AND NOT in title, key words or abstract: china OR taiwan OR japan OR asia OR asian OR korea

\section{Inclusion and exclusion criteria}

Inclusion criteria for studies were that they must be written in English, focus on an older population (aged 65 and older or with an average age above 70 years), include people with a formal diagnosis of dementia, use a validated tool for assessing prescribing appropriateness and characterise PIP, and report prevalence of PIP as an outcome. To manage the scope of this study, we focused the review on studies based on populations from Western Europe, North America and Australia. This also ensured studies included were based on countries with comparable health services to the UK. As our main objective was the retrieval of prevalence estimates, qualitative analyses and literature reviews were excluded.

\section{Study selection}

The titles retrieved from a search of bibliographic databases were screened and duplicates removed. Potentially relevant titles were selected for abstract screening; these were then further selected for full-text analysis. The 
full-text analysis produced the final list of manuscripts included in this review. The review process was undertaken by two researchers working independently (JD and $\mathrm{KB})$. No significant discrepancies were identified between the two compiled lists. Screening and review processes, as well as data extraction, were completed using Microsoft Office Excel (2013).

\section{Data extraction and analysis}

Data extraction began by identifying general characteristics of the studies, that is, year, country of origin, study design and clinical setting of the study. Next, we summarised results including population size and characteristics as well as prevalence of PIP (ie, percentage) and classification criteria. We also extracted estimates for statistical associations for level of polypharmacy (ie, ORs), and for level of comorbidity and for specific comorbidities. We also extracted the drugs related to the PIP criteria identified in each study (eg, hypnotics and sedative, laxative or analgesics). We calculated prevalence of PIP using random-effect meta-analysis models for each clinical setting. Due to clinical heterogeneity between studies, these were the best estimates we could derive, but they should not be taken as accurate prevalence rates. I-squared analysis was used to test for heterogeneity. The Appraisal tool for Cross-Sectional Studies (AXIS tool), providing a systematic assessment of each study based on 20 components, was used to assess the quality of the evidence (online supplementary eTable1). ${ }^{32}$

\section{RESULTS}

The initial search of bibliographic databases yielded 273 citations, 221 of which remained after removing 52 duplicates (figure 1). Title screening identified 44 studies that were potentially relevant and retained for abstract screening. Of these, 20 studies were selected for full-text analysis, and 12 studies were deemed eligible for this review (table 1). Of the 12 studies included, nine were published in the past 5 years. The majority of the studies

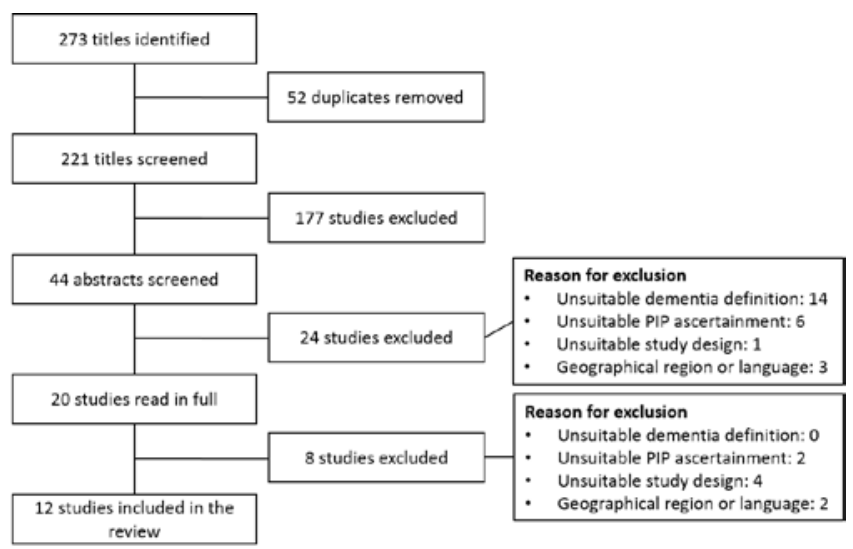

Figure 1 Selection process for peer-reviewed journal articles (Preferred Reporting Items for Systematic Reviews andMeta-Analyses flow chart). PIP, potentially inappropriate prescribing. $(n=8)$ were based in Europe, with the remaining studies based in North America ( $n=2)$ and Australia $(n=2)$.

\section{Prevalence of PIP}

All 12 studies included in this review present estimates of PIP prevalence, although focusing on different settings (four community, four nursing/care home, two mixed, two providing separate estimates for community and nursing/care home patients). There was significant variation between studies, with high clinical heterogeneity and estimates of prevalence ranging from $13.9 \%$ to $64.4 \%$. The lowest value of $13.9 \%$ was identified by a study focussing only on individuals with mild dementia. Prevalence was lower in individuals living in the community with a pooled prevalence of $31 \%$ (95\% CI 9 to 52); reported estimates varied from $13.9 \%$ to $64.4 \%$. Prevalence of PIP for individuals in nursing homes and specialised care homes was higher with a pooled estimate of $42 \%$ (95\% CI 30 to 55 ); the lowest recorded prevalence was $26.9 \%$ and the highest $54.9 \%$ (figure 2). The two studies providing independent estimates for both groups showed similar results (figure 2). Prevalence in studies not differentiating between care settings was $38 \%$ (95\% CI 17 to 59) with the lowest at $24.4 \%$ and highest at $60.0 \%$.

\section{Methods to assess PIP}

In the 12 studies identified, there were 9 different toolkits used to identify PIP, of which 8 were non-dementiaspecific toolkits designed for older individuals in general and one was a dementia-specific toolkit. ${ }^{21}$ The dementia-specific toolkit was used in two studies focusing on nursing home patients, although one study complemented it with additional criteria for drug-disease and drug-drug interactions. PIP prevalence for studies using the dementia-specific criteria was $26.9 \%$ and $53.9 \%$, respectively. The most common set of generalist criteria used was the STOPP/START criteria, applied in three studies.

\section{Drugs frequently reported as the cause of PIP}

The drugs related to the specific PIP criteria were described in all but one study. ${ }^{33}$ Here, we summarise the most frequently reported drugs related to PIP in the included studies. Reported medications included those likely related to dementia (sedative and hypnotics $(\mathrm{n}=10)$, antipsychotics $(\mathrm{n}=4)$, cholinesterase inhibitors $(n=4)$ and antidepressants $(n=3)$. However, PIPs related to drugs used to treat comorbidities of dementia were also identified, including cardiovascular drugs and antihypertensives $(\mathrm{n}=8)$, non-steroidal anti-inflammatory drugs (NSAID; $n=4)$, antiacid drugs $(n=3)$, laxatives $(n=3)$, antihistamine drugs $(n=2)$, diabetes drugs $(n=2)$, anti-incontinence including antimuscarinic drugs $(n=2)$, analgesic drugs including opioids $(\mathrm{n}=1)$, antibiotic drugs $(\mathrm{n}=1)$ and antiepileptic dugs $(\mathrm{n}=1)$. Lastly, PIP related to high anticholinergic burden was identified in nine studies. 


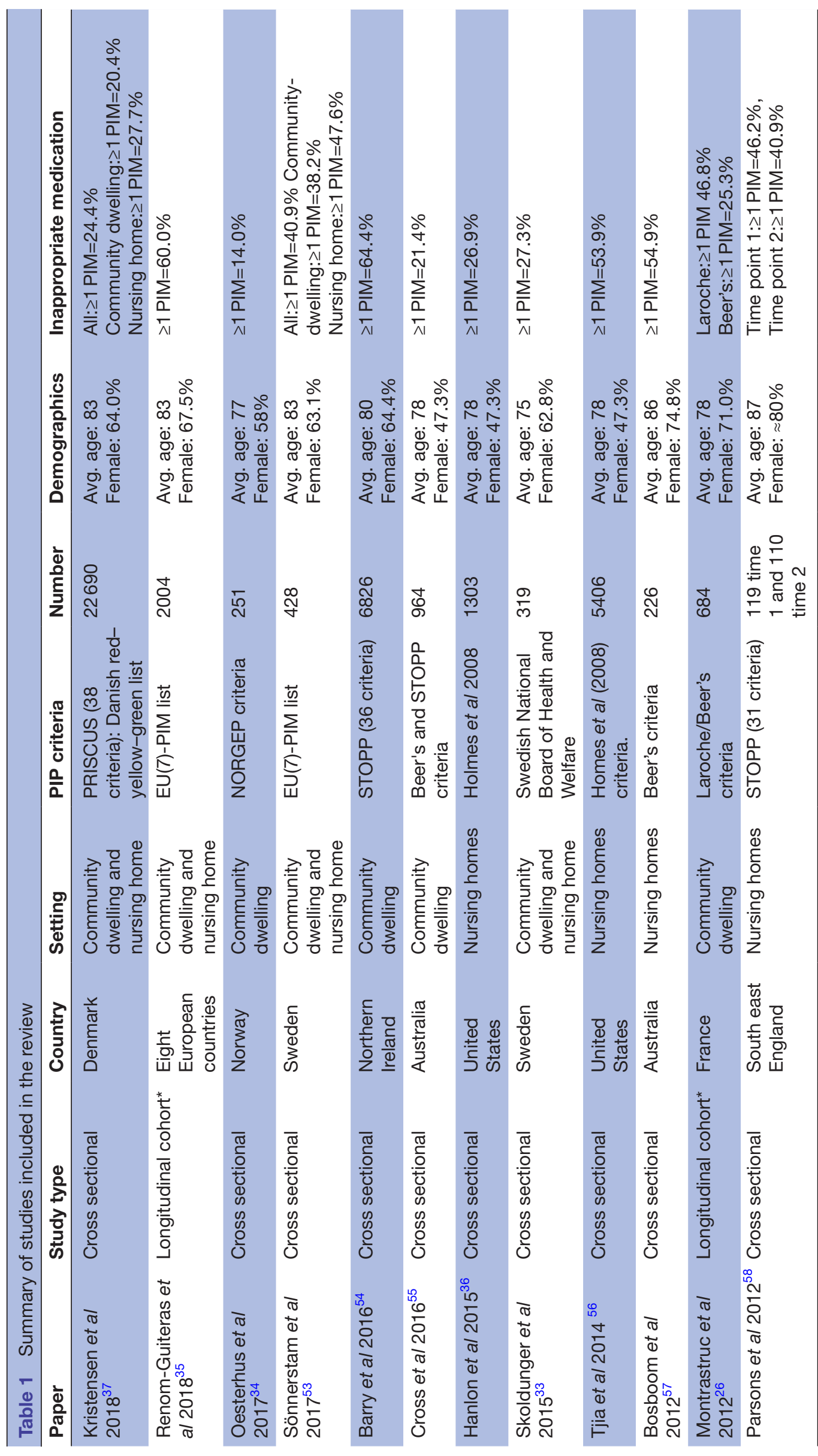




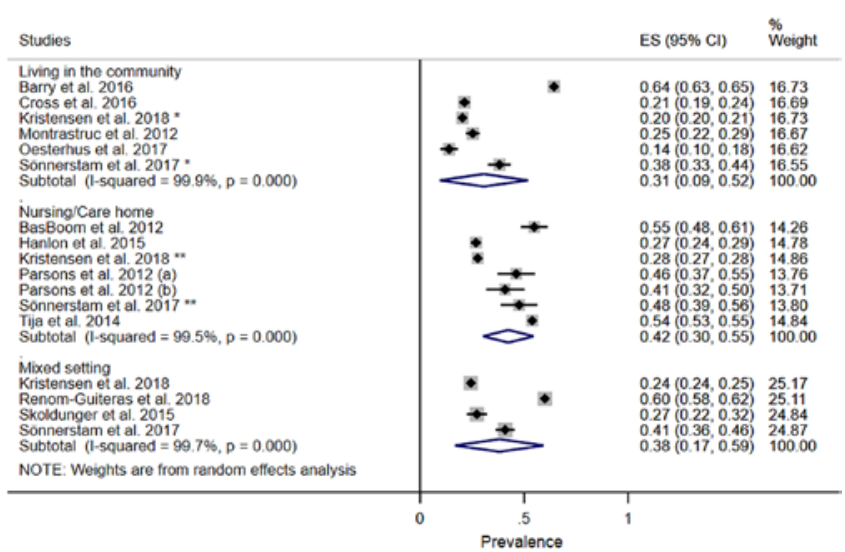

Figure 2 Pooled prevalence of inappropriate prescribing in people with dementia. Studies were grouped by study setting. *Subsample living in the community. ${ }^{*}$ Subsample living in nursing home. (a) Diagnosed with mild dementia. (b) Diagnosed with severe dementia. (c) Time point 1. (d) Time point 2.

\section{Comorbidities}

Four of the 12 studies provided a measure of the burden of comorbidity in the individuals studied (table 2). Three studies used a defined score system to measure the individual level of general comorbidity; Oesterhus et al used the Cumulative Illness Rating Scale, ${ }^{34}$ Renom-Guiteras et al used the Charlson Comorbidity index (CCI $)^{35}$ and Hanlon et al 2015 used the CCI excluding dementia ${ }^{36}$ (table 2). Of these, two studies describe a positive association with PIP (OR 1.35, 95\% CI 1.03 to 1.77 and OR $1.51,95 \%$ CI 1.30 to 1.75 ) while the third study did not describe a statistical association (figure 3). A fourth study, Skoldunger et al 2015, separated the CCI score into four categories, and described an increase in prevalence of PIP alongside an increasing comorbidity score $(0=9.5 \%$, $1=15.9 \%, 2=16.6 \%, 3-34=26.4 \%) .^{33}$

Only one study compared PIP prevalence in individuals with and without specific conditions, identifying excess prevalence rates in individuals diagnosed with diabetes $(+7.6 \%)$, hypertension $(+8.7 \%)$ and depression

Table 2 Factors associated with potentially inappropriate prescribing identified in the studies included in this review.

\begin{tabular}{lll}
\hline Paper & Comorbidities & Polypharmacy \\
\hline Kristensen et al $2018{ }^{37}$ & Community: polypharmacy ( $\geq 5$ prescriptions) OR 1.50 \\
& (1.45-1.55); excessive polypharmacy ( $\geq 10$ prescriptions) \\
& OR 1.51 (95\% Cl 1.44 to 1.58$)$ \\
& Nursing home polypharmacy OR $0.88(95 \% \mathrm{Cl} 0.84$ to \\
& $0.92) ;$ excessive polypharmacy OR 0.68 (95\% Cl 0.65 to
\end{tabular}

Renom-Guiteras et al Comorbidity Charlson (0-2 vs 3-34): OR 1.35 $2018^{35} \quad(95 \% \mathrm{Cl} 1.03$ to 1.77 , p-value 0.029).

Oesterhus et al $2017^{34} \quad$ Cumulative Illness Rating Scale (range 0-52): Polypharmacy ( $\geq 5$ prescriptions) $45 \%$ and psychotropic OR 1.51 (95\% Cl 1.30 to 1.75$)$. polypharmacy ( $\geq 3$ prescriptions) $2.8 \%$. Number of medications: OR 1.50 (95\% CI 1.29 to $1.73, \mathrm{p}<0.001)$.

Sönnerstam et al $2017^{53}$

Barry et al $2016^{54}$

Cross et al $2016^{55}$

Polypharmacy ( $\geq 4$ prescriptions): OR 7.6 (95\% Cl 6.6 to 8.7).

Polypharmacy ( $\geq 5$ prescriptions) and hyperpolypharmacy ( $\geq 10$ prescription) were associated with high PIM prevalence

\begin{tabular}{|c|c|c|}
\hline Hanlon et al $2015^{36}$ & $\begin{array}{l}\text { Charlson Comorbidity Index (excluding } \\
\text { dementia: range 0-33): OR } 1.39 \text { ( } 95 \% \text { Cl } 0.97 \\
\text { to } 2.00)\end{array}$ & \\
\hline Skoldunger et al $2015^{33}$ & $\begin{array}{l}\text { PIM prevalence by Charlson Comorbidity } \\
\text { Index level }(0=9.5 \%, 1=15.9 \%, 2=16.6 \% \text {, } \\
3-34=26.4 \%)\end{array}$ & $\begin{array}{l}\text { PIM prevalence by number of prescriptions }(0-1=0.8 \% \text {, } \\
2-4=8.6 \%, \geq 5=29.6 \%) \text {. }\end{array}$ \\
\hline Tjia et al $2014^{56}$ & $\begin{array}{l}\text { Difference in PIP prevalence by diagnosis: } \\
\text { diabetes+7.6\%, hypertension+8.7\%, } \\
\text { depression+8.3\%, stroke+0.8\%, heart failure } \\
-0.7 \% \text { and osteoporosis: }-4.6 \% \text {. }\end{array}$ & \\
\hline \multicolumn{3}{|l|}{ Bosboom et al $2012^{57}$} \\
\hline Montrastruc et al $2012^{26}$ & & $\begin{array}{l}\text { Association PIP with polypharmacy: OR } 3.6(95 \% \text { CI } 2.6 \\
\text { to } 4.5) \text {. }\end{array}$ \\
\hline Parsons et al $2012^{58}$ & & $\begin{array}{l}\text { Correlation between number of medicines prescribed and } \\
\mathrm{PIP}=0.335(\mathrm{p}<0.01)\end{array}$ \\
\hline
\end{tabular}

$p$ values included when available in the original publication.

PIP, potentially inappropriate prescribing. 
Studies

Renom-Guiteras et al. 2018 (a)

Oesterhus et al. 2017 (b)

Hanlon et al. 2015 (c)

Hanlon et al. 2015 (c) **
ES $(95 \% \mathrm{Cl})$

$1.35(1.03,1.77)$

$1.51(1.30,1.75)$ $1.39(0.96,2.00)$

2
Figure 3 Summary of the analysis of the association between increasing levels of comorbidity and incidence of potentially inappropriate prescribing. (a) Charlson Comorbidity Index (<3 vs $\geq 3$ ). (b) Cumulative Illness Rating Scale (0-52). (c) Charlson Comorbidity Index (0-32). *Mild-tomoderate dementia. ${ }^{* \star}$ Severe dementia.

$(+8.3 \%)$, but the inverse for osteoporosis $(-4.6 \%)$ (table 2).

\section{Dementia versus free from dementia}

Two studies compared the prevalence of PIP in individuals diagnosed with dementia against those living free from dementia (table 1). Both identified higher prevalence of PIP in people living with dementia. Skoldunger et al. (2015) reported a PIP prevalence in individuals diagnosed with dementia of $27.3 \%$ vs $11.8 \%$ in individuals with no dementia, $\mathrm{p}<0.001) .{ }^{33}$ Kristensen et al reported higher prevalence compared with those with no dementia in individuals diagnosed with dementia living in the community $(20.4 \%$ vs $12.5 \%, \mathrm{p}<0.001)$, but the opposite for those in nursing home settings $(27.7 \%$ vs $33.7 \%, \mathrm{p}<0.001)$. $^{37}$

\section{DISCUSSION}

Our state-of-the-art review examines the prevalence of PIP among older individuals living with dementia drawing on evidence published between 2007 and 2018, summarises current trends in PIP prevalence, and identifies key gaps in understanding of the role of comorbidities in increasing risk of PIP. There are considerable differences in PIP prevalence rates reported for different care settings and measured by different PIP criteria. Prevalence estimates vary greatly between studies, even within those focusing on similar clinical settings. The included studies provide a broad overview of the current understanding of the state of prescribing for people living with dementia. Studies published in the past decade have identified a high proportion of PIP in individuals diagnosed with dementia. In line with studies focusing on the older population generally, comorbidity and polypharmacy were associated with increased prevalence of PIP (table 2). ${ }^{37-39}$ PIP prevalence was higher in nursing/ care homes compared with community settings, although with overlapping confidence intervals, driven by possible higher levels of comorbidity and polypharmacy.

The drugs most frequently identified as related to PIP were also similar to those identified in studies focussing on the general older population. ${ }^{38} 39$ These can be categorised in two broad groups. A first group includes hypnotics, sedatives, and antidepressants (ie, benzodiazepines and tricyclic-antidepressants), drugs that may be a bigger driver of PIP in people diagnosed with dementia compared with those without, as these are often used to control physical aggression, wandering and sleep disturbances. ${ }^{40}$ The same rationale is also applicable to antipsychotics, as increased likelihood of antipsychotic prescription is associated with in-patient settings, diagnosis of vascular or Parkinson's disease dementia and greater severity of dementia. ${ }^{41}$ However, a second group was also identified which included a variety of PIP instances related to medications used to treat comorbidities of dementia. PIP related to medication used to treat cardiovascular disease, including hypertension, was identified in eight studies. The studies also identified PIP related to drugs used to treat incontinence and constipation, and to combat infection and manage pain, as well as non-steroidal anti-inflammatory drugs and anti-histamines. This suggests that treatments to manage comorbidities of dementia are also significant drivers of PIP in this group. Importantly, PIP associated with drugs causing high anti-cholinergic burden was identified in nine studies. Drugs with high anti-cholinergic burden include drugs commonly used to treat dementia symptoms (ie, sedatives) and drugs used to treat comorbidities of dementia (ie, anti-arrhythmic and anti-muscarinic drugs). ${ }^{42}$

In people living with dementia comorbidities are managed pharmacologically, often resulting in patients being prescribed a large number of medications. ${ }^{43} 44$ The frequent use of medication in people living with dementia in combinations that have been known to cause adverse reactions suggests that these individuals are especially at risk of inappropriate prescribing,. ${ }^{42245}$ In fact Maidment et al suggest that dementia drives the presence of risk factors for adverse drug reactions. ${ }^{46}$ Fox et al proposed that dementia dominates clinical encounters, reducing attention to other comorbidities and thus increasing the chances of inadequate treatment and inappropriate prescribing. ${ }^{47}$ The studies included in this review suggest that people diagnosed with dementia are more susceptible to PIP than those not diagnosed. Only two studies compared the two groups, but both found higher prevalence rates in individuals diagnosed with dementia. ${ }^{33} 37$ However, in Kristensen et al higher prevalence of PIP in those diagnosed with dementia was found only in those living in the community, with lower prevalence for those in nursing/care homes. ${ }^{37}$

The identified prevalence of PIP in people diagnosed with dementia was high, ranging from $13.9 \%$ to $64.5 \%$. There is a need to optimise medicines use in this group. The presence of multi-morbidities and associated polypharmacy has long been described as one of the drivers, if not the main driver, of PIP in these age groups. ${ }^{42} 4648-50$ This is supported by the findings from this review, as 3 out of the four studies measuring comorbidity showed a positive association with PIP prevalence. ${ }^{33-36}$ However, research on the specific comorbidities, or combinations of comorbidities, driving the risk of inappropriate prescribing remains sparse with only one study providing PIP estimates for selected comorbidities. 
Understanding which comorbidities are associated with higher prevalence of PIP, and how this affects outcomes (eg, ADR) would be a significant next step in optimising treatment of people living with dementia. People living with dementia, particularly those in advanced stages, are often excluded from trials. ${ }^{27} 28$ As a result, existing evidence for the pharmacological management of chronic conditions (eg, diabetes) does not fully reflect the needs of people with dementia, nor does it provide conclusive evidence that it improves health outcomes or quality of life. ${ }^{4451} 52$ A 2018 systematic review on management of chronic conditions in people with dementia identified only six studies, covering depression, osteoporosis, diabetes and cardiovascular disease. ${ }^{44}$ Of these, the impact of treatment in clinical outcomes was reported for only one condition. ${ }^{44}$ The reduced representation of people with more severe dementia in clinical trials is particularly problematic as treatment targets for comorbidities of dementia may need to change as dementia progresses. ${ }^{29}$ Further work is necessary to identify the medical conditions driving increased risk of PIP. Identifying the comorbidities, or combination of comorbidities, of dementia that increase risk of PIP can support clinical practice by characterising patient profiles of individuals particularly at risk of PIP that may benefit from a comprehensive geriatric assessment or pharmacological review. Additionally, this could help to prioritise those comorbidities where medical research can have the greatest impact in optimising medication, and improving health outcomes and quality of life, for people living with dementia.

This state-of-the-art review of studies published in the last 10 years indicates that PIP remains a significant issue for people living with dementia, and that the presence of additional comorbidities contributes to the exacerbation of this issue. It remains unclear which specific comorbidities drive risk of PIP. Such information has the potential to inform clinicians and other medical professionals about individuals at high risk of PIP. Moreover, in the absence of clinical evidence on treatment of comorbidities for people with dementia, understanding which comorbidities place individuals at greater risk of PIP can help define priorities for future medical research.

\section{Strengths and limitations}

A key strength of this review is the structured approach used to identify relevant studies. We used a set of defined search terms applied to the most widely used databases (eg, MEDLINE, EMBASE and Compendex), ensuring repeatability of the results. The selection process was carried out by two reviewers working independently. In doing so, we aimed to make selection and review a robust process and the inclusion criteria aimed to ensure we identified studies with transparent methodology and sound design. These criteria included a requirement for a formal diagnosis of dementia, validated tools for classification of PIP and clearly stated PIP prevalence.

This review has some limitations. Focusing on the past 10 years of research means we may have excluded important studies published before 2007; however, scrutiny of literature reviews analysed when preparing and executing this review suggests that major findings in the area of PIP and dementia have not been excluded by focusing on this period. ${ }^{4131522}$ Second, by searching only two databases it is possible some relevant studies were missed. The included studies displayed significant clinical heterogeneity, likely arising from different study designs. While we used methods of estimating prevalence for each clinical setting that account for this heterogeneity, we emphasise that the estimates produced should be viewed as a guide that can stimulate discussion and practice development, and not as precise estimates of prevalence. Future reviews focusing on providing more accurate estimates of prevalence rates and relative risks of PIP should adopt more stringent criteria for inclusion.

\section{CONCLUSIONS}

Potentially inappropriate prescribing is common in people living with dementia, with PIPs reported related to drugs used to manage dementia symptoms but also to drugs used to treat comorbidities. This review has highlighted that comorbidity and polypharmacy are associated with increased prevalence of PIP in people with dementia, but the specific conditions driving the increase in risk remain unknown. Identifying the comorbidities driving risk of PIP can facilitate targeting of interventions to reduce PIP in people with living with dementia. Further work is necessary to investigate the role of comorbidities in causing PIP and the effects on clinical outcomes.

\section{Twitter Joao Delgado @delgadojpc}

Contributors JD was responsible for study concept and design, acquisition of data, analysis and interpretation of data, and preparation of the manuscript. KB was responsible for acquisition of data, analysis and interpretation of data, and preparation of the manuscript. LC was responsible for study concept and design and preparation of the manuscript.

Funding This work was supported by Alzheimer's Society (grant number: 338 (AS-JF-16b-007)). The sponsor played no role in the design, methods, subject recruitment, data collection, analysis or preparation of the paper.

Competing interests None declared.

Patient consent for publication Not required.

Provenance and peer review Not commissioned; externally peer reviewed.

Data availability statement All data relevant to the study are included in the article or uploaded as supplementary information.

Open access This is an open access article distributed in accordance with the Creative Commons Attribution Non Commercial (CC BY-NC 4.0) license, which permits others to distribute, remix, adapt, build upon this work non-commercially, and license their derivative works on different terms, provided the original work is properly cited, appropriate credit is given, any changes made indicated, and the use is non-commercial. See: http://creativecommons.org/licenses/by-nc/4.0/.

ORCID iD

Joao Delgado http://orcid.org/0000-0003-1648-871X

\section{REFERENCES}

1 Swanson KA, Carnahan RM. Dementia and comorbidities: an overview of diagnosis and management. J Pharm Pract 2007;20:296-317. 
2 Langa KM, Foster NL, Larson EB. Mixed dementia. JAMA 2004;292. [Internet].

3 Knapp M, Guerchet M, McCrone M, et al. Dementia UK: update. Alzheimer's Society, 2014

4 Meloni E, Vetrano DL, Bernabei R, et al. Appropriateness of pharmacological treatment in older people with dementia. Ital J Med 2015:9:212-6.

5 Melzer D, Delgado J, Winder R, et al. The Age UK almanac of disease profiles in later life (August 2015) [Internet]. Age UK. Exeter, United Kingdom; 2015. 1-68. Available from: http://www.ageuk.org. uk/professional-resources-home/research/reports/health-wellbeing/ almanac-disease-profiles/

6 Molander L, Gustafson Y, Lövheim H. Longitudinal associations between blood pressure and dementia in the very old. Dement Geriatr Cogn Disord 2010;30:269-76.

7 Poblador-Plou B, Calderón-Larrañaga A, Marta-Moreno J, et al. Comorbidity of dementia: a cross-sectional study of primary care older patients. BMC Psychiatry 2014;14:1-8. [Internet].

8 Zuliani G, Galvani M, Sioulis F, et al. Discharge diagnosis and comorbidity profile in hospitalized older patients with dementia. Int $J$ Geriatr Psychiatry 2012;27:313-20.

9 Callahan CM, Schubert CC. The complexities of comorbidity in dementia. Nat Rev Neurol 2014;10:184-6. [Internet].

10 Kanagaratnam L, Mahmoudi R, Novella J-L, et al. Adverse drug reactions in elderly subjects hospitalized in a specialized dementia management unit. Drugs Aging 2014;31:769-76.

11 Barry HE, Cooper JA, Ryan C, et al. Potentially inappropriate prescribing among people with dementia in primary care: a retrospective cross-sectional study using the enhanced prescribing database. J Alzheimers Dis 2016:1-11. [Internet]

12 Moriarty F, Hardy C, Bennett K, et al. Trends and interaction of polypharmacy and potentially inappropriate prescribing in primary care over 15 years in Ireland: a repeated cross-sectional study. BMJ Open 2015:5:e008656-8.

13 Johnell K. Inappropriate drug use in people with cognitive impairment and dementia: a systematic review. Curr Clin Pharmacol 2015;10:178-84. [Internet].

14 Hamilton HJ, Gallagher PF, O'Mahony D. Inappropriate prescribing and adverse drug events in older people. BMC Geriatr 2009;9:1-4

15 Patel T, Slonim K, Lee L. Use of potentially inappropriate medications among ambulatory home-dwelling elderly patients with dementia: a review of the literature. Can Pharm J 2017;150:169-83.

16 Wallace E, McDowell R, Bennett K, et al. Impact of potentially inappropriate prescribing on adverse drug events, health related quality of life and emergency Hospital attendance in older people attending general practice: a prospective cohort study. J Gerontol Ser A Biol Sci Med Sci [Internet] 2016;00:glw140.

17 Lucchetti G, Lucchetti ALG. Inappropriate prescribing in older persons: a systematic review of medications available in different criteria. Arch Gerontol Geriatr 2017;68:55-61. [Internet].

18 Spinewine A, Schmader KE, Barber N, et al. Appropriate prescribing in elderly people: how well can it be measured and optimised? The Lancet 2007;370:173-84.

19 Fick DM, Semla TP, Steinman M, et al. American geriatrics Society 2019 updated AGS beers Criteria ${ }^{\circledR}$ for potentially inappropriate medication use in older adults. J Am Geriatr Soc 2019;67:674-94.

20 O'Mahony D, O'Sullivan D, Byrne S, et al. STOPP/START criteria for potentially inappropriate prescribing in older people: version 2. Age Ageing 2014;44:213-8.

21 Holmes HM, Sachs GA, Shega JW, et al. Integrating palliative medicine into the care of persons with advanced dementia: identifying appropriate medication use. J Am Geriatr Soc 2008;56:1306-11.

22 Parsons C. Polypharmacy and inappropriate medication use in patients with dementia: an underresearched problem. Ther Adv Drug Saf 2017;8:31-46.

23 Opondo D, Eslami S, Visscher S, et al. Inappropriateness of medication prescriptions to elderly patients in the primary care setting: a systematic review. PLoS One 2012;7:e43617.

24 Tommelein E, Mehuys E, Petrovic M, et al. Potentially inappropriate prescribing in community-dwelling older people across Europe: a systematic literature review. Eur J Clin Pharmacol 2015;71:1415-27.

25 Browne J, Edwards DA, Rhodes KM, et al. Association of comorbidity and health service usage among patients with dementia in the UK: a population-based study. BMJ Open 2017;7:e012546-8.

26 Montastruc F, Gardette V, Cantet C, et al. Potentially inappropriate medication use among patients with Alzheimer disease in the REAL. FR cohort: be aware of atropinic and benzodiazepine drugs! Eur $J$ Clin Pharmacol 2013;69:1589-97.
27 Banzi R, Camaioni P, Tettamanti M, et al. Older patients are still under-represented in clinical trials of Alzheimer's disease. Alzheimer's Res Ther [Internet] 2016;8:1-10.

28 Taylor JS, DeMers SM, Vig EK, et al. The disappearing subject: exclusion of people with cognitive impairment and dementia from geriatrics research. J Am Geriatr Soc 2012;60:413-9.

29 Page AT, Potter K, Clifford R, et al. Medication appropriateness tool for co-morbid health conditions in dementia: consensus recommendations from a multidisciplinary expert panel. Intern Med $\mathrm{J}$ 2016;46:1189-97.

30 Grant MJ, Booth A. A typology of reviews: an analysis of 14 review types and associated methodologies. Health Info Libr J 2009;26:91-108.

31 Dudas R, Malouf R, McCleery J, et al. Antidepressants for treating depression in dementia. Cochrane Database of Systematic Rev 2018;2:1-94

32 Downes MJ, Brennan ML, Williams HC, et al. Development of a critical appraisal tool to assess the quality of cross-sectional studies (axis). BMJ Open 2016;6:e011458-7.

33 Sköldunger A, Fastbom J, Wimo A, et al. Impact of inappropriate drug use on hospitalizations, mortality, and costs in older persons and persons with dementia: findings from the SnAC study. Drugs Aging 2015;32:671-8.

34 Oesterhus R, Aarsland D, Soennesyn $\mathrm{H}$, et al. Potentially inappropriate medications and drug-drug interactions in homedwelling people with mild dementia. Int J Geriatr Psychiatry 2017;32:183-92.

35 Renom-Guiteras A, Thürmann PA, Miralles R, et al. Potentially inappropriate medication among people with dementia in eight European countries. Age Ageing 2018;47:68-74. [Internet].

36 Hanlon JT, Aspinall SL, Handler SM, et al. Potentially suboptimal prescribing for older veteran nursing home patients with dementia. Ann Pharmacother 2015:49:20-8.

37 Kristensen RU, Nørgaard A, Jensen-Dahm C, et al. Polypharmacy and potentially inappropriate medication in people with dementia: a nationwide study. J Alzheimers Dis 2018;63:383-94.

38 Ble A, Masoli JAH, Barry HE, et al. Any versus long-term prescribing of high risk medications in older people using 2012 beers criteria: results from three cross-sectional samples of primary care records for 2003/4, 2007/8 and 2011/12. BMC Geriatr 2015;15:146.

39 Sura SD, Carnahan RM, Chen H, et al. Prevalence and determinants of anticholinergic medication use in elderly dementia patients. Drugs Aging 2013;30:837-44. [Internet].

40 Rochon PA, Vozoris N, Gill SS. The harms of benzodiazepines for patients with dementia. Can Med Assoc J 2017;189:E517-8.

41 Barnes TRE, Banerjee S, Collins N, et al. Antipsychotics in dementia: prevalence and quality of antipsychotic drug prescribing in UK mental health services. Br J Psychiatry 2012;201:221-6.

42 Richardson K, Fox C, Maidment I, et al. Anticholinergic drugs and risk of dementia : case-control study. Br Med J 2018;360:1-12.

43 Blass DM, Black BS, Phillips $\mathrm{H}$, et al. Medication use in nursing home residents with advanced dementia. Int J Geriatr Psychiatry 2008;23:490-6.

44 Page A, Etherton-Beer C, Seubert LJ, et al. Medication use to manage comorbidities for people with dementia: a systematic review. J Pharm Pract Res 2018;48:356-67.

45 Ramsey CM, Gnjidic D, Agogo GO, et al. Longitudinal patterns of potentially inappropriate medication use following incident dementia diagnosis. Alzheimers Dement 2018;4:1-10.

46 Maidment ID, Fox C, Boustani M, et al. Medication managementthe missing link in dementia interventions. Int J Geriatr Psychiatry 2012;27:439-42.

47 Fox C, Smith T, Maidment I, et al. The importance of detecting and managing comorbidities in people with dementia? Age Ageing 2014; $43: 741-3$

48 Biessels GJ, Staekenborg S, Brunner E, et al. Risk of dementia in diabetes mellitus: a systematic review. Lancet Neurol 2006;5:64-74.

49 Hamilton H, Gallagher P, Ryan C, et al. Potentially inappropriate medications defined by STOPP criteria and the risk of adverse drug events in older hospitalized patients. Arch Intern Med 2011;171:1013-9.

50 Bunn F, Burn A-M, Goodman C, et al. Comorbidity and dementia: a scoping review of the literature. BMC Med 2014;12:192. [Internet].

51 Bunn F, Goodman C, Reece Jones P, et al. What works for whom in the management of diabetes in people living with dementia: a realist review. BMC Med 2017;15:1-18.

52 van der Wardt V, Logan P, Conroy S, et al. Antihypertensive treatment in people with dementia. J Am Med Dir Assoc 2014;15:620-9.

53 Sönnerstam E, Sjölander M, Gustafsson M. An evaluation of the prevalence of potentially inappropriate medications in older people 
with cognitive impairment living in Northern Sweden using the EU(7)PIM list. Eur J Clin Pharmacol 2017;73:735-42.

54 Barry HE, Cooper JA, Ryan C, et al. Potentially inappropriate prescribing among people with dementia in primary care: a retrospective cross-sectional study using the enhanced prescribing database. JAD 2016;52:1503-13. [Internet].

55 Cross AJ, George J, Woodward MC, et al. Potentially inappropriate medications and anticholinergic burden in older people attending memory clinics in Australia. Drugs Aging 2016;33:37-44.
56 Tjia J, Rothman MR, Kiely DK, et al. Daily medication use in nursing home residents with advanced dementia. J Am Geriatr Soc 2010;58:880-8.

57 Bosboom PR, Alfonso H, Almeida OP, et al. Use of potentially harmful medications and health-related quality of life among people with dementia living in residential aged care facilities. Dement Geriatr Cogn Dis Extra 2012;2:361-71. [Internet].

58 Parsons $\mathrm{C}$, Johnston S, Mathie E, et al. Potentially inappropriate prescribing in older people with dementia in care homes: a retrospective analysis. Drugs Aging 2012;29:143-55. [Internet]. 